\title{
Research on Safety Assessment Method for Bridge Structure Based on Variable Weight Synthesis Method Yongfeng $\mathrm{Xu}^{1, \mathrm{a}}$ Hailong Wang ${ }^{1, \mathrm{~b}}$ Liqun Zhang $^{1, \mathrm{c}}$ \\ ${ }^{1} \mathrm{HeBei}$ Institute of Architecture and Civil Engineering ahh-0002@163.com, ${ }^{\text {b }}$ wanghl@hebiace.edu.cn, ${ }^{\text {czhanliqun5133@163.com }}$
}

Key words: variable weight synthesis method; bridge structure; safety assessment

Abstract. Variable weight synthesis method is a safety assessment method which is using the varied weight principle, base on analytic Hierarchy Process and combining the theory of Grey relevancy, it reduce influence that secondary cause is not obvious in assessment process, and make the result more correct and dependable. In the paper, variable weight principle and the assessment method were expounded detailedly.

\section{Variable weight principle and Variable weights method}

\subsection{Variable weight principle}

In the literature "The application of variable weight synthesis method in estimate of real estate investment environment", variable weight synthesis method is the definition of:

1) Assume that a m-dimensional vector, $W^{0}=\left(W_{1}^{0}, W_{2}^{0}, \ldots, W_{m}^{0}\right)$, if present $W_{j}^{0} \in(0,1)$ makes $\sum_{j=1}^{m} W_{J}^{0}=1$ correct for arbitrary variables $j \in\{1,2, \ldots m\}$;

2) Given a m-d vector of parts keep weight $W(X) \Delta\left[W_{1}(X) 2 W_{1}(X), \ldots, W_{m}(X)\right]$, for given mapping: $W:[0,1]^{m} \rightarrow(0,1]$, it makes that:

(1) $\sum_{j=1}^{m} W_{j}(X)=1$

(2)Assume that $\alpha_{j}, \beta_{j} \in[0,1]$, and $\alpha_{j} \leq \beta_{j}$, for arbitrary variables $j \in\{1,2, \ldots . m\}$, present $W_{j}$ in regard to $x_{j}$ in the internal $\left[0, \alpha_{j}\right]$ and $\left.\left[\beta_{j}, 1\right]\right]$ respectively decrease and increase progressively.

3)Assume that the vector $S(X) \Delta\left[S_{1}(X), S_{2}(X), \ldots, S_{m}(X)\right]$ is a m-d vector of parts variable weight, for the given mapping $S:[0,1]^{m} \rightarrow(0,+\infty)^{m}$, for arbitrary variables $j \in\{1,2, \ldots m\}$, assume $\alpha_{j}, \beta_{j} \in[0,1]$, and $\alpha_{j} \leq \beta_{j}$ present that:

(1)For arbitrary variables $j \in\{1,2, \ldots m\}$ for fixed weights vector $W^{0}=\left(W_{1}^{0}, W_{2}^{0}, \ldots, W_{m}^{0}\right)$, $W_{j}(X)=\frac{W_{j}^{0} S_{j}(X)}{\sum_{k=1}^{m} W_{k}^{(0)} S_{k}(X)}$ in the internal $\left[0, \alpha_{j}\right]$ and $\left.\left[\beta_{j}, 1\right]\right]$ respectively decrease and increase progressively.

(2)If $0 \leq x_{i} \leq x_{k} \leq \alpha_{i} \wedge \alpha_{k}, \quad S_{i}(x) \geq S_{k}(x)$, and $\beta_{i} \vee \beta_{k} \leq x_{i} \leq x_{k} \leq 1$, then

$$
\frac{w^{0} \cdot S(x)}{\sum_{j=1}^{m} w_{j}^{0} S_{j}(x)} \square\left\{\frac{w_{1}^{0} \cdot S_{1}(x)}{\sum_{j=1}^{m} w_{j}^{0} S_{j}(x)}, \frac{w_{2}^{0} \cdot S_{2}(x)}{\sum_{j=1}^{m} w_{j}^{0} S_{j}(x)}, \ldots \frac{w_{m}^{0} \cdot S_{m}(x)}{\sum_{j=1}^{m} w_{j}^{0} S_{j}(x)}\right\}
$$

be called m-d vector of parts variable weight 。 


\subsection{Amendment of variable weights method}

In the variable weights method, when synthesis assessment the assessed value of hundred-mark system, it will result data with denominator larger, and produce larger round-off error. For convenience of calculations and considering the required precision, to transform hundred-mark system into percentage of system, and then, by adjusting coefficients in the formulas, getting the synthesis assessed value of hundred-mark system. in addition, for some sequences of inspection data, it is impossible to collect all the data, but it is needed to compare the curve of sequences of inspection data, so in this paper, considering base on the grey theory related principle to offset the malconformation and incomprehensive of data. The paper make the improvement.

$$
V=\sum_{j=1}^{m} 100 w_{j}\left(x_{1}, \cdots, x_{m}, w_{1}^{(0)}, \cdots, w_{m}^{(0)}\right) x_{j}
$$

In formula: $V$ is the assessed value;

$$
\begin{aligned}
& w_{j}^{(0)} \text { is initial weight value of the jth indicators, and } \sum_{j=1}^{n} w_{j}^{(0)}=1 ; \\
& w_{j} \text { is weight value after variable weight; } \\
& x_{j} \text { is the percentage of assessment value of the jth indicators. }
\end{aligned}
$$

And

$$
w_{j}\left(x_{1}, \cdots, x_{n}, w_{1}^{(0)}, \cdots, w_{n}^{(0)}\right)=\frac{w_{j}^{(0)} \frac{\partial B\left(x_{1}, \cdots, x_{n}\right)}{\partial x_{j}}}{\sum_{k=1}^{n} w_{k}^{(0)} \frac{\partial B\left(x_{1}, \cdots, x_{n}\right)}{\partial x_{k}}}
$$

In formula, $B\left(x_{1}, \cdots, x_{n}\right)$ is balanced function, according to experience of engineering practice, the balanced function is:

$$
B\left(x_{1}, \cdots, x_{n}\right)=\sum_{j=1}^{n} x_{j}^{\alpha} \quad(0<\alpha \leq 1)
$$

simultaneous equations (3) and (4), it can get:

$$
w_{j}\left(x_{1}, \cdots, x_{n}, w_{1}^{(0)}, \cdots, w_{n}^{(0)}\right)=\frac{w_{j}^{(0)} x_{j}^{\alpha-1}}{\sum_{k=1}^{n} w_{k}^{(0)} x_{k}^{\alpha-1}}
$$

SO

$$
V=\sum_{j=1}^{m} \frac{100 w_{j}^{(0)} x_{j}^{\alpha}}{\sum_{k=1}^{n} w_{k}^{(0)} x_{k}^{\alpha-1}}
$$

$\alpha$ : balance coefficient, according to experience of engineering practice, $\alpha=0.2$, it can meet most of engineering situation.

The inspection data of bridge can be divided into three categories: the first kind is non-numeric data with describing the state of bridge components only or dividing grade simply; the second is a single numerical; and the third is a data series. it is needed that applies dimensionless method to the third kind data, compare and analyze the two curves of data series. Introduce grey correlativity analysis and calculate non-uniform variation coefficient to get the assessment result, the formula as follows. 


$$
V=\sum_{j=1}^{m} \frac{100 w_{j}^{(0)} x_{j}^{\alpha}}{\sum_{k=1}^{n} w_{k}^{(0)} x_{k}^{\alpha-1}} r\left(x_{0}, x_{i}\right)
$$

And $r\left(x_{0}, x_{i}\right)$ calculate according to the formula as below[5][8]:

$$
r\left(X_{0}, X_{i}\right)=\frac{1}{\mathrm{n}-1} \sum_{k=1}^{n-1}\left[1+\left|\frac{a^{(1)}\left(x_{0}(k+1)\right)}{x_{0}(k+1)}-\frac{a^{(1)}\left(x_{i}(k+1)\right)}{x_{i}(k+1)}\right|\right]^{-1}
$$

\section{Advantage of variable weight synthesis method}

Compared with constant weight synthesis method, variable weight synthesis method can make the influence of components with damage badly more prominent. In structure, maybe individual component damage can be lead to the bridge operational be limited, even impact to the safety of the bridge. Compared with the result of constant weight synthesis method and variable weight synthesis method as below.

Table1 assess table of a bridge

\begin{tabular}{|c|c|c|c|}
\hline indicator & weight & Technical status & explain \\
\hline pier and abutment & 0.31 & 80 & better \\
\hline girder & 0.13 & 80 & better \\
\hline pylon & 0.14 & 75 & normal \\
\hline stay cable & 0.37 & 80 & better \\
\hline subsidiary facilities & 0.04 & 5 & failure badly \\
\hline
\end{tabular}

Using the data in table1, assessment the health of bridge with variable weight synthesis method and constant weight synthesis method, the result as below.

1)constant weight synthesis method:

$$
B C I_{2}=80 \times 0.31+80 \times 0.13+74 \times 0.14 \times 80 \times 0.37+5 \times 0.04=75.51>66
$$

The conclusion is "qualified";

2)variable weight synthesis method( $\alpha=0.2)$ :

$$
\begin{aligned}
& \sum_{k=1}^{m} w_{k}^{(0)} x^{\alpha-1}=\sum_{k=1}^{9} w_{k}^{(0)} x_{k}^{-0.8}=0.31 \times 0.80^{-0.8}+0.13 \times 0.80^{-0.8}+0.14 \times 0.75^{-0.8}+0.37 \times 0.80^{-0.8} \\
& +0.04 \times 0.05^{-0.8}=1.5840 \\
& \quad \text { score } V_{2}: \\
& V_{2}=\sum_{j=1}^{m} \frac{100 w_{j}^{(0)} x_{j}^{(\alpha-1)}}{\sum_{k=1}^{n} w_{k}^{(0)} x_{k}^{(\alpha)}}=\frac{100}{1.584}(0.2965+0.1243+0.1322+0.3539+0.0220) \\
& =58.64
\end{aligned}
$$

The conclusion is "disqualified".

The different conclusion with the different value of $\alpha$ is show in table2. The value of $\alpha$ reflect tolerance of local damage, with the increase of $\alpha$, tolerance increase. 
Table2 consultable table of equal coefficient

\begin{tabular}{|c|c|c|c|c|c|}
\hline equal coefficient & $\alpha=0$ & $\alpha=0.2$ & $\alpha=0.5$ & $\alpha=0.8$ & $\alpha=1.0$ \\
\hline Score of girder & 49.52 & 58.64 & 68.59 & 74.19 & 75.51 \\
\hline
\end{tabular}

By the above examples, it can be got that when the subsidiary facilities damage badly, the result of constant weight synthesis method is "qualified" while variable weight synthesis method is "disqualified". It is show that variable weight synthesis method can well reflect the local defect and considering the malconformation into assessment. It is more tally with the actual situation.

\section{Application example of method}

The inspection data of line shape control about abridge is in table 3.

Table3 1evel evaluation of reference point

\begin{tabular}{|c|c|c|c|c|}
\hline $\begin{array}{c}\text { coordinate } \\
(\mathrm{m})\end{array}$ & $\begin{array}{c}\text { designed elevation } \\
\left(\mathrm{X}_{0}\right)\end{array}$ & $\begin{array}{c}\text { measured elevation } \\
\left(\mathrm{X}_{\mathrm{i}}\right)\end{array}$ & $\begin{array}{c}\text { Error } \\
(\mathrm{m})\end{array}$ & $\begin{array}{c}\text { assessed value } \\
\left(\mathrm{D}_{\mathrm{i}}\right)\end{array}$ \\
\hline 0 & 86.550 & 86.538 & 0.012 & 90 \\
\hline 20 & 86.844 & 86.830 & 0.014 & 90 \\
\hline 40 & 87.079 & 87.056 & 0.023 & 80 \\
\hline 60 & 87.247 & 87.235 & 0.012 & 90 \\
\hline 80 & 87.432 & 87.419 & 0.013 & 90 \\
\hline 100 & 87.534 & 87.520 & 0.014 & 90 \\
\hline 120 & 87.541 & 87.517 & 0.024 & 80 \\
\hline 140 & 87.531 & 87.508 & 0.023 & 80 \\
\hline 160 & 87.371 & 87.357 & 0.014 & 90 \\
\hline 180 & 87.168 & 87.153 & 0.015 & 90 \\
\hline 200 & 86.993 & 86.977 & 0.016 & 90 \\
\hline 220 & 86.774 & 86.752 & 0.022 & 80 \\
\hline 240 & 86.537 & 86.526 & 0.011 & 90 \\
\hline & & & & \\
\hline
\end{tabular}

According to formula (5), calculate variation of weight show as fig.1 as below. 


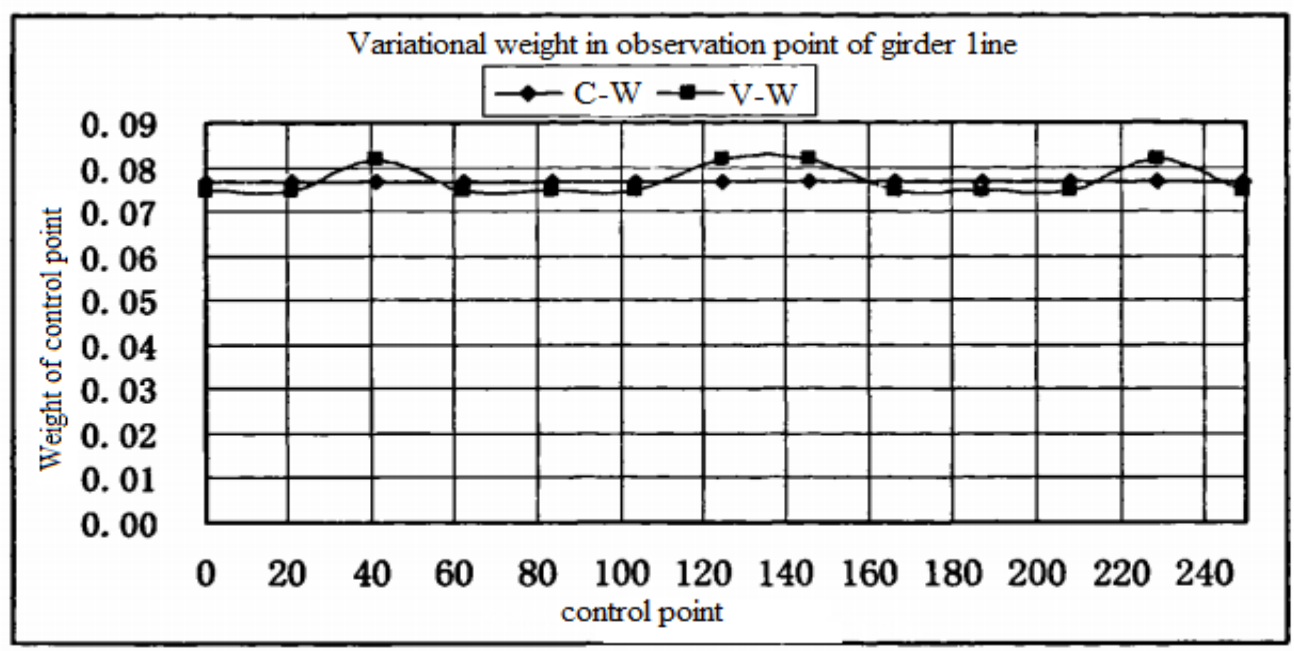

C-W:constant weight synthesis method; V-Wrvariable weight synthesis method

Fig.1 Variational weight in observation point of girder 1ine

According to formula(7)and(8)calculate as below:

$$
V=\sum_{j=1}^{m} \frac{100 w_{j}^{(0)} x_{j}^{\alpha}}{\sum_{k=1}^{n} w_{k}^{(0)} x_{k}^{\alpha-1}} r\left(x_{0}, x_{i}\right)=84.14
$$

$x_{k}=D_{i} / 100, D_{i}$ : assessed value. $w^{(0)}=1 / n(\mathrm{n}=13)$ initial weight value, See table3-1.

$$
\begin{gathered}
r\left(X_{0}, X_{i}\right)=\frac{1}{\mathrm{n}-1} \sum_{k=1}^{n-1}\left[1+\left|\frac{a^{(1)}\left(x_{0}(k+1)\right)}{x_{0}(k+1)}-\frac{a^{(1)}\left(x_{i}(k+1)\right)}{x_{i}(k+1)}\right|\right]^{-1}=0.968 \\
X_{0}=\left[X_{0}(1), X_{0}(2), \cdots X_{0}(13)\right] \text { designed elevation; } \\
X_{i}=\left[X_{i}(1), X_{i}(2), \cdots X_{i}(13)\right] \quad \text { measured elevation. }
\end{gathered}
$$

Assessment score of girder alignment is 84.14 , contrast table4, the bridge can be classified grade 1.

Table4 The reference standard of bridge technique condition evaluation

\begin{tabular}{|c|c|c|}
\hline Score & Grade & Explain \\
\hline $80<V \leq 100$ & Grade 1 & Well, only daily maintenance \\
\hline $60<V \leq 80$ & Grade 2 & Normal, daily maintenance and minor repair \\
\hline $40<V \leq 60$ & Grade 3 & Weak, repair and load test \\
\hline $20<V \leq 40$ & Grade 4 & Bad, Stop running and strengthen \\
\hline $0<V \leq 20$ & Grade 5 & Danger, considering dismantle and rebuild \\
\hline
\end{tabular}

Note: the evaluation standard is made according to "Evaluation procedure for bearing capacity of highway bridge”.

\section{Conclusion and prospect}

The article explains the variable weight synthesis method, and apply the method into the process of bridge assessment. Though it has the rationality of the method, but it still exist some problem. First, although the assessment apply new method, but most of the index still reference codes and specifications and previous research results, it needs to do a a more in-depth discussion. Second, the initial weight value used in the paper, it is still the average weight which has certain blindness, it 
needs to do a more in-depth discussion, too.

\section{References}

[1] Bai Yunchao The application of variable weight synthesis method in estimate of real estate investment environment, Journal of Adult Education College of Hebei University, 2007.09 Volume 9, 3rd Issue

[2] Gao Huaizhi, Wang Junjie, A review of research and application of detective and evaluation technology for bridges, World Information On Earthquake Engineering , 2000, 16(2)

[3] Li Rong, Research on method of bridge health fuzzy comprehensive evaluation based on the analytic hierarchy process, Hunan University, 2007

[4] Zhang Yongqing, Feng Zhongju, . To evaluate the bridge safety by AHP, Journal of Xi'an Highway University, 2001, 21(3)

[5] Lan Hai, Shi Jiajun, . Degree of Grey Incidence and Variable Weight Synthesizing Applied in Bridge Assessmen. JOURNAL OF TONGJI UNIVERSITY , 2001, 29(l)

[6] Wu Huacheng, Xiang Yiqiang, . Assessment the tension and the linetype state cable2 stayed bridge based on variable weight synthesis, 1995.4, 16.

[7] Xiang Yiqiang, Wu Huacheng, Research on condition assessment system of long-span prestressed concrete cable-stayed bridge [J]. Journal of China \& Foreign Highway, 2005, 25(5)

[8]Liu Sifeng, Dang Yaoguo, Fang Zhigeng, The grey system theory and application [M]. Beijing: Science publishing house, 2004. 